\title{
ESTADO SITUACIONAL Y JUSTIFICACIÓN DE ÉTICA DE LA INVESTIGACIÓN
}

\author{
SITUATIONAL CONDITION AND ETHICS RESEARCH GROUNDS
}

JUAN PABLO RODRÍGUEZ MIRANDA

Profesor asociado, Universidad Distrital Francisco José de Caldas, Facultad del Medio Ambiente y Recursos Naturales. Director del grupo de investigación AQUAFORMAT, jprodriguezm@udistrital.edu.co

\section{VIDAL FERNANDO PEÑARANDA GALVIS}

Profesor asociado, Universidad Distrital Francisco José de Caldas, Facultad del Medio Ambiente y Recursos Naturales. Coordinador Proyecto Curricular de Tecnología en Saneamiento Ambiental, vfpenarandag@udistrital.edu.co

\section{RESUMEN:}

Este artículo expone los elementos conceptuales del estado, la situación y la justificación de la ética en el desarrollo de la investigación, teniendo en cuenta que los actos, la voluntad, sus propuestas y sus fines de investigación, son por esencia racionalidades que el ser humano puede orientar. La voluntad es dependiente de lo que se quiere o no, de su estado íntimo y racional; así mismo voluntad-libertad, como punto principal de los actos son necesarios y de utilidad, propios y únicos en la vida, por sus intenciones nunca libres de la prevención, del conocimiento y de sus resultados.

\section{PALABRAS CLAVE:}

Ética, investigación.

\section{ABSTRACT:}

This article presents the conceptual elements of state, situation and justification of ethics in research, taking into account that acts and will, its proposals and research purposes, essentially are rationalities than human been can direct. The will depends on you want or not; its depends on intimate and rational state too; likewise will-freedom, as main aspect of acts are both necessary and useful, unique in our own life, their intentions never free of prevention, knowledge and results.

\section{KEYWORDS:}

Ethics, research. 
ARTE \& DISEÑ0, ISSN: 1692-8555, Vol. 11 N² 2, Julio - Diciembre 2013

JUAN PABLO RODRÍGUEZ MIRANDA - VIDAL FERNANDO PEÑARANDA GALVIS tica e investigación son dos acepciones imprescindibles como herramientas de aporte meritorio y responsable en el proceso investigativo. Al asumirlas en la práctica y sin reserva, la humanidad superó las barreras del oscurantismo educativo y liberó en lo académico y social el verdadero razonamiento considerable para la cognición de ser, y para la implícita normatividad en el progreso del mundo moderno. Al universo científico le concierne observar e indagar sobre el permanente cambio para la adaptación de las circunstancias que rodean a este mundo plausible. El no alejarse de los parámetros conceptuales es lo que le ha permitido aclarar el papel que le corresponde como investigador y juez integrador del equilibrio en la naturaleza, con consideración de las conductas adoptadas por las comunidades. Para los diversos ámbitos de ser, con la responsabilidad de prevenir, en lo que es la vida actual, la sociedad ha tenido alejamientos que en obligación le concierne valorar para evitar sucesos contradictorios. La misma ética debe llevar a la advertencia, con las posibles razones de contingencia que consiente para el estudio situacional y en las que contribuye la investigación.

La ética fortalece a la sociedad y va de la mano con el escudriñamiento; en su reflexión, la colectividad, con sus caracteres y diferencias, se torna inflexible y justificada para aprestar y afrontar las intemperancias del futuro. Pero la ética en una profesión es la obligación de una conducta correcta. Las múltiples situaciones a las que hay que dar respuesta desde cada profesión muestran que la ética profesional es una parte de cada acto profesional individual que incluye un conflicto entre el efecto intencionado y el efecto conseguido. Así pues, desde el punto de vista de la investigación, un acto ético es el que se ejerce responsablemente, evitando el perjuicio a personas, que a veces se realiza inconscientemente, por estar vinculado el daño a los métodos que el investigador utiliza para la consecución de sus fines (Buendía \& Berrocal de Luna, 2001, p.2). Las necesidades y acontecimientos de la civilización siempre originarán cambios en los que amerita enfilar los instrumentos transformadores que dan el soporte en ciencia y tecnología por medio de la investigación. Pero la metodología para resolver problemas, y la complejidad de situaciones que surgen al plantearlos, más los elementos que se incorporan como producto de la formación epistemológica y psicología del investigador, pueden presentar más dificultades que hay que asumir y resolver con la particularidad de los mismos métodos de prueba y comprobación. Con referencia a Popper, Sabino (1996) expone que "...la mayoría de los buenos investigadores y muchos de los excelentes, no son otra cosa que hombres largamente educados en la disciplina de la ciencia, gente que se "ha hecho" investigadora mediante la voluntad y el estudio y cuya tarea es la de "proponer teorías y ponerlas a prueba" (PP. 51 - 52). Los investigadores, en su minuciosidad integral de indagación, deciden con su experiencia teórica y práctica evitar menos controversia y discusión. En cambio, de este criterio y, sin el margen de especulación, en el campo de estudio y del conocimiento para el enfoque directo actual, sí se debe esclarecer con puntualidad los problemas fundamentales en la evolución de comprobar más allá de lo que se sabe. Al establecer la naturaleza de la investigación, su delimitación y concreción del problema también se deben instaurar los beneficios, los criterios de evaluación técnica, teórica-ideológica y su puesta experimental de cobertura útil y participación práctica en la protección de la humanidad y de lo que la rodea.

Además, se debe determinar qué es lo importante por investigar, para que justifique su accionar con su sistema de valores y prioridades integrales. En esa lógica del orden con sus marcos racionales, del desarrollo, los paradigmas, las técnicas y los métodos, lo importante es resolver los problemas (entre otros, conseguir que una mayoría obtenga un empleo digno y pueda conseguir el pan diario). Así como la investigación no está exenta de normas y de la ideología del investigador, tampoco ninguna investigación puede quedar al margen de necesidades comunes que aquejan a la humanidad; este es, en realidad, junto con lo ambiental, los problemas crecientes por abordar como prioridad universal.

En investigación, los modelos son considerados como métodos sujetos a los procesos y a las variables, pero también son de versátil aplicación para asumirlos o no como indicadores fiables en la obtención de resultados, dependientes del tema y de la experiencia del investigador. No obstante en la unidad del método científico, su aplicación depende, en gran medida, del asunto: esto explica la multiplicidad de técnicas y la relativa independencia de los diversos sectores de la ciencia (Bunge, 2002, p.25). Lo anterior, en el plano de las inferencias, estas difieren en esa misma expresión en técnica y desarrollo, en lo que respecta al objetivo, método y alcance, con el dilema del antiguo dualismo filosófico materia-espíritu, razón-experiencia.

\section{DESARROLLO ESTRUCTURA Y CONTENIDOS DE CONTROL}

En el estudio de la ética de la investigación es importante analizar cada contexto y las modalidades de interdependencia con el cuerpo de conocimientos que se posee. La planificación no es solo un acto técnico que posee una alta responsabilidad, en el cual se entiende que el mejor método es poner en práctica la conducta correcta, proyectar el mínimo impacto en el campo investigado, con la focalización de proteger las especies y todo lo que existe. Al evitar perjuicios entre la intención y lo conseguido ya hay una clara restricción de los problemas en la aplicación del campo ético con el subconjunto profesional general, más con el explícito enfoque de que se deben resolver los interrogantes para llegar al acuerdo.

En la correlación ética e investigación se caracterizan los niveles de decisión y estrategia operacional, que involucran la 
ARTE \& DISEÑO, ISSN: 1692-8555, Vol. 11 N² 2, Julio - Diciembre 2013 Estado situacional y justificación de ética en la investigación, págs. 39 - 43

racionalidad, concretar los planes a nivel global, la multiplicidad de actores, la complejidad del proyecto, en el proceso de identificación del problema, implementación, delimitación, metodología. No obstante, en lo elemental de no explicitar en la formulación una rigurosa guía, sí definir bien la intención que se propone, y posicionar junto con la evaluación, los juicios, la valoración de alternativas y soluciones. Asimismo, son diferentes los códigos de ética, con sus acuerdos desde Venecia, Núremberg, Helsinki, Naciones Unidas, Colombia 2001.

En síntesis de otros elementos y patrones para tratar también son múltiples los problemas éticos fundamentales, inherentes a los planes junto con los métodos que los investigadores utilizan para su orientación y valores. Se comprende que al aplicar las normas y costumbres, con la intención que fuere en la investigación educativa, debe ser un acto ético, conscientes de que la naturaleza de la investigación es trasparente, con mínima invasión a los actores, poca alteración en lo abordado y alta participación de los beneficios. Si encontráramos al azar un grupo de científicos, aun dentro de un mismo campo o disciplina, encontraríamos que sus ideas sobre un problema determinado difieren, a veces de modo radical (Jaramillo, 1995, p.26). Se puede argumentar que en el desarrollo teórico, los rasgos éticos con los criterios y valores de los hechos explícitos, dan las diferentes opciones para debatir y tomar la decisión en las variables. Definida la situación ética, en su extensión estratégica y racional, el universo la asimila en su orientación y con sus principios constituyen la base de todo el orden disciplinar, para la Nación, el abogado, el juez, el religioso, el político, el maestro, el Estado, los gobiernos, con la diferenciación entre personas, cosas y seres que no se pueden suplir.

En el mundo actualmentr se habla de posturas como ética de la investigación, ser perceptivos, fijarnos mucho y reflexionar para ser mejores, encajar en la sociedad y aportarle con todas las complejidades, para vivir con calidad de vida y como humano eficiente. Ética es comprender qué comportamientos nos convienen, saber que tenemos que expirar, pero la impronta se debe caracterizar con la planificación de saber que no todo da igual. Para definir el acto humano de la voluntad libre y deliberada: es decir, no todo lo que el hombre hace o ejecuta es un acto humano, o sea que no toda la actividad del hombre es objeto material de la ética (Hortta \& Rodríguez, 1994, p.148).

Si las observaciones están bien planteadas, al analizar y asociar los fenómenos con los hechos presentes y del pasado, las deducciones y resultados serán análogos con la relación y objeto del juicio. En el principio esencial para llegar a una cercana hipótesis es ineludible recopilar todos los elementos que se relacionan, por lo tanto, se deben comparar y circunscribir a los hechos con fenómenos anteriores, sin que lleguen a ser una sola reflexión o afirmación de juicio irrefutable. En lo instrumental e ilustrativo de estos conceptos de deducción y exploración de la intuición, resultados y observaciones, como líneas de conducta que se deben formular claras, al incorporarlas al análisis y cir- cunstancias de los sucesos, en el contenido siempre debe estar presente el conocimiento con sus leyes e instrucciones, como la síntesis del pensamiento y su solución. Para Bergson, una de las primeras características de la intuición consiste en que se opone a la inteligencia, ya que esta no puede tener acceso a los secretos de las cosas y de la vida (Baudouin, 2006, p.35).

En lo fundamental de la investigación científica están los contenidos teóricos y prácticos; estos se asocian en lo conceptual con los métodos y técnicas que usualmente se emplean en el proceso, con sus dimensiones y etapas, las cuales no solo pertenecen al plano metodológico. En toda crítica real del objeto de estudio interviene el ánimo del investigador; ese espíritu en su asociación de ideas es el principio del sentido común, y en su forma más sintética de selección, se le asimila el razonamiento ético. Entre otras circunstancias del desarrollo, en su referencia de análisis y curiosidad, agrupan la observación, la indagación y la crítica, para una mayor explicación real y satisfactoria de conocimiento del fenómeno en su campo de estudio.

Las diversas y crecientes realidades de la formación y el conocimiento se conciben desde varias áreas específicas del discernimiento y la inventiva; el área de estudio con su acorde empleo sistemático de los elementos conceptuales básicos complementan el requisito para la investigación, y en su similitud, estos hechos los requerirá y analizará en sus circunstancias el profesional investigador.

El espíritu del conocimiento, la creación y la indagación se van adquiriendo con las etapas formativas; en su naturaleza interior se estudiarán con el ambiente y las costumbres; varían con sus posibilidades, sus referentes y contextos. De otra parte, al recopilar la información y asimilar los contenidos se debe tener claro el propósito y los objetivos para orientar la práctica investigativa, con sus métodos e instrumentos básicos. En la situación corriente de este estudio de acercamiento, para tratar lo profundo del conocimiento de una forma más sencilla, en su continuidad de línea de interés, el hábito de pensar permite extraer las deducciones correspondientes de estas observaciones y el sentido común concluye (Tashi, 2000).

En la inicial aproximación a la metodología general de la investigación científica, el profesional, con los contenidos adquiridos en su disciplina, más los elementos de estudio, precisión conceptual y técnicos, debe estar en capacidad académica de proyectar el diseño investigativo y el tratamiento estadístico. En la posición intelectual de perfilar y emprender los proyectos que exige el plantear y resolver los determinados problemas se aplica la lógica de probabilidades, y en consecuencia, la abstracción de los temas básicos, para garantizar los resultados con el seguimiento de los métodos.

Por razones como las anteriores y según el propósito, debe profundizar en las consultas fidedignas y la bibliografía, en los contenidos relacionados y los trabajos existentes. Intercambiar conceptos con los expertos, tanto en los puntos no frecuentes como también coincidentes, para la formulación del problema, 
ARTE \& DISEÑ0, ISSN: 1692-8555, Vol. 11 N² 2, Julio - Diciembre 2013

JUAN PABLO RODRÍGUEZ MIRANDA - VIDAL FERNANDO PEÑARANDA GALVIS

las hipótesis, metodología y desarrollo, más la suma de los planes que conlleva la pesquisa. No todos los autores se rigen por un patrón exclusivo y rígido de indagación; hay quienes por su recurrencia investigativa y práctica, por su habilidad en escribir consideran que no existe un procedimiento único, ni una regla universal en la exploración.

\section{EL ACTO VOLITIVO ÉTICO}

La voluntad y la libertad en el entendimiento son dos condiciones específicas para expresar los propósitos de los actos y su intención:

1. El rito dogmático de ir al oficio religioso es, en definitiva, un acto libre, espiritual y humano que se adquiere por convicción del alma, y para el pensar general no le hace mal a nadie. Algunas personas lo han sopesado, lo han puesto en práctica, con su realización y orientación de conducta se han beneficiado en lo psíquico durante toda su vida.

2. El señor o la señora, por la premura de la hora, salió y dejó la estufa prendida al calentar un café. Olvidó un acto, entre el físico-fisiológico y el espiritual, dos actos de vieja data. Sobrevino una conflagración.

3. En conclusión, la omisión de uno de los actos no es buena, sin embargo, la intención sí lo ha sido. El juicio variaría si hubiese apagado la estufa.

La voluntad es dependiente de lo que se quiere o no, de su estado íntimo y racional, asimismo voluntad-libertad, como punto principal de los actos, son necesarios y de utilidad, propios y únicos en la vida, por sus intenciones nunca libres de la prevención, del conocimiento y de sus resultados. Las personas y las familias no pierden su individualidad ni su independencia por asumir un credo; además hay una tendencia en las costumbres a seguir las tradiciones de la sociedad con sus recuerdos y regulación. Por lo tanto, un ejemplo de esta afirmación es que la ciudad era una confederación: en religión siguió subsistiendo una muchedumbre de pequeños cultos, sobre los cuales se estableció un culto común; en política siguió funcionando una multitud de pequeños gobiernos, y sobre ellos se estableció un gobierno común (De Coulanges, 2002, p.144).

\section{ÉTICA Y EXIGENCIAS GENERALES DE LA INVESTIGACIÓN CIENTIIFICA}

Por la razón que fuere, de los elementos cognoscitivos que configuran los actos humanos para apropiar el saber, en su realización principal del describir, la ética y la ciencia no deben estar exentas de lo que se quiere conocer, realizar y cuál es su fin con la implícita naturaleza de hacer el bien. Probar es la disposición del conocimiento para cuando se empieza un estudio, por cuanto se presume que estan en consideración su tratado con la mente y la determinación de los utensilios y medios que se van a emplear. En ciencia y su perspectiva son importantes los fundamentos del aprehender, y si en esa misma intención de exigencia es conveniente o posible su obrar, por la deliberación de los actos de responsabilidad y/o por temores de fuerzas externas en contra de la libertad, las facultades, la razón y sus consecuencias son causas en sí mismas de la realización de sus actos y en derivación someterse a su juicio.

Estudiar los compendios del conocimiento con el objeto material de la ética y sus actos de lo que se va a realizar, el obrar bien hacia la naturaleza, del ser humano y todas las demás cosas, es la exigencia del saber qué se requiere en la medida del avance tecnológico, de cómo son las vicisitudes para buscar la perfección y corregir el error.

Está en las propias manos humanas la destreza de emprender el estudio de sus actos, en la responsabilidad de la investigación científica y su aplicabilidad reside el corregir su esencia y de todos los demás bienes para controlar la suerte de su futuro ambiental y de su vida.

Las sociedades inofensivas tienen la misión de descubrir la reflexión ética, cuestionar y ser tolerantes; su razón de bien mayor y riqueza están fundamentados por los derechos, no por formas compungidas de pensar por correspondencia económica, eliminar la envidia y actuar con decoro, precavidos de no caer en "lo malo de las roscas es no pertenecer a ellas" (Peñaranda, 2012, p.99).

\section{CONCLUSIONES}

Por toda la actividad reflexiva, de las capacidades de ser de cada persona, su voluntad e importancia de investigar, de realizar propuestas, más el alcance en las consecuencias del hacer el bien o su negación, está el conocer y enterarse del porqué, de la realidad y para qué lo hace; sus situaciones comunes del dinamismo lo hacen un ser capaz e individual de evolucionar y transformar todo lo que esté a su alcance.

Los actos y la voluntad, con sus propuestas y sus fines de investigación, son por esencia racionalidades que el ser humano puede dirigir en sus conductas. El mundo, entre la realidad y su trascendencia de apariencias, con todas sus características de proyección y conocimiento siempre le interesará indagar sobre mecanismos de perfeccionamiento. Como una advertencia más de la significación del descubrir, emitirá juicios, elaborará y propondrá fines, no como condición de capricho o de fin último, sino como máxima razón y, en consecuencia, de esta posesión surgirán los bienes más grandes que son la libertad, la felicidad, y en la ineludible convivencia con la naturaleza no cesará en el ideal de alcanzar la excelencia para una mejor calidad de vida. Ética e investigación son objetos formales y radicales de la ciencia; estos dos actos de la personalidad proporcionan el más alto grado de criterio y elevación para sopesar el bien y el 
mal en la transversalidad de realizar las situaciones comunes que conciernen al universo y su coexistencia.

\section{REFERENCIAS BIBLIOGRÁFICAS}

Baudouin, B. (2006). Las Claves de la Intuición. Barcelona: Editorial De Vecchi.

Buendía E, \& Berrocal L, E. (2014, 25 de abril). La Ética de la Investigación Educativa. Universidad de Granada [en línea]. Disponible en: (http://www.uhu.es/agora/version01/digital/numeros/01/01 articulos/miscelanea/buendia.PDF).

Bunge, M. (2002). La Ciencia, su Método y su Filosofía. Bogotá, D. C.: Panamericana Editorial Ltda.

Coulanges, F. de. (2002). La Ciudad Antigua. (p.144) Bogotá D.C.: Panamericana Editorial.

Jaramillo, L. (1995). Módulo 1 (P. 26). Serie: Aprender a investigar. Bogotá D.C.: División de Procesos Editoriales de la Secretaría General del ICFES. Serie: Aprender a investigar. Módulo 1, p. 26.

Hortta, E. de J \& Rodríguez G, V. (1994). Ética General. (p.148). Colombia: Editorial Presencia.

Peñaranda, V, F. (2012). Viaje por la Ética. Bogotá D.C.: Universidad Distrital Francisco José de Caldas.

Sabino, C, A. (1996). El proceso de Investigación. Medellín, (Colombia): Editorial Cometa de papel.

Tashi, Y. (2000). El sentido común. Bogotá D.C: Ediciones Universales.

\section{FORMA DE CITAR ESTE ARTÍCULO}

Rodríguez Miranda, Juan Pablo y Peñaranda Galvis, Vidal Fernando (2013). Estado situacional y justificación de ética en la investigación. Revista Arte y Diseño Facultad de Arquitectura, Arte y Diseño, Universidad Autónoma del Caribe, Barranquilla. ISSN 1692- 8555 Vol. 11 (N.2) P.P 39- 43 